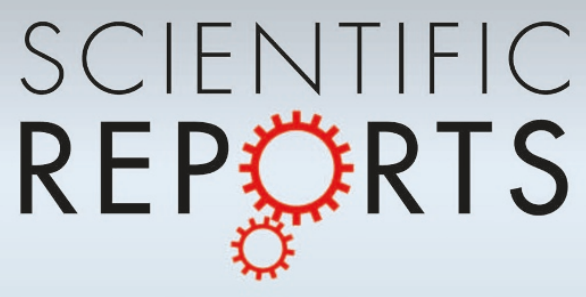

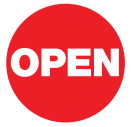

SUBJECT AREAS: CONDENSED MATTER PHYSICS

QUANTUM FLUIDS AND SOLIDS THEORETICAL PHYSICS

ELECTRONIC PROPERTIES AND MATERIALS

Received

27 June 2012

Accepted

23 November 2012

Published

18 December 2012

Correspondence and requests for materials should be addressed to S.S. (sorella@sissa.it) or S.Y. (yunoki@riken.

ip)

\title{
Absence of a Spin Liquid Phase in the Hubbard Model on the Honeycomb
} Lattice

\author{
Sandro Sorella $a^{1,2,3}$, Yuichi Otsuka ${ }^{3}$ \& Seiji Yunoki ${ }^{3,4,5}$
}

\begin{abstract}
${ }^{1}$ SISSA - International School for Advanced Studies, Via Bonomea 265, 34136 Trieste, Italy, ${ }^{2}$ Democritos Simulation Center, CNR IOM Instituto Officina dei Materiali, 34151 Trieste, Italy, ${ }^{3}$ Computational Materials Science Research Team, RIKEN AICS, Kobe, Hyogo 650-0047, Japan, ${ }^{4}$ Computational Condensed Matter Physics Laboratory, RIKEN ASI, Saitama 351-0198, Japan, ${ }^{5}$ CREST, Japan Science and Technology, Kawaguchi, Saitama 332-0012, Japan.
\end{abstract}

A spin liquid is a novel quantum state of matter with no conventional order parameter where a finite charge gap exists even though the band theory would predict metallic behavior. Finding a stable spin liquid in two or higher spatial dimensions is one of the most challenging and debated issues in condensed matter physics. Very recently, it has been reported that a model of graphene, i.e., the Hubbard model on the honeycomb lattice, can show a spin liquid ground state in a wide region of the phase diagram, between a semi-metal (SM) and an antiferromagnetic insulator (AFMI). Here, by performing numerically exact quantum Monte Carlo simulations, we extend the previous study to much larger clusters (containing up to 2592 sites), and find, if any, a very weak evidence of this spin liquid region. Instead, our calculations strongly indicate a direct and continuous quantum phase transition between SM and AFMI.

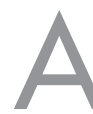

spin liquid can be considered as a Mott insulator that is not adiabatically connected to any band insulator and does not break any symmetry even at zero temperature. Recently, much attention has been focused on a possible spin liquid in two or three spatial dimensions ${ }^{1-5}$. On one hand, it has been suggested experimentally that several organic materials represent good candidates for spin liquids $s^{6-8}$. On the other hand, the existence of a spin liquid has so far been demonstrated only in very few and particularly simplified models ${ }^{9,10}$.

In order to understand this issue, let us consider a model Hamiltonian on a lattice describing the insulating state of electrons at half-filling, i.e., one electron per lattice site. Since the charge gap is assumed, only spin degrees of freedom remain and can be described by the spin-1/2 Heisenberg model. Since any spin-1/2 model corresponds to a well defined interacting hard core boson model, the crucial question is to understand how - at zero temperature - these bosonic degrees of freedom can avoid Bose-Einstein condensation and/or crystallization, necessary conditions for a stable spin liquid with no long-range order of any kind.

In this report, we study the ground state of the half-filled Hubbard model on the honeycomb lattice (see Fig. 1) defined by the following Hamiltonian,

$$
\hat{H}=-t \sum_{\langle i, j\rangle, \sigma}\left(c_{i \sigma}^{\dagger} c_{j \sigma}+c_{j \sigma}^{\dagger} c_{i \sigma}\right)+U \sum_{i} n_{i \uparrow} n_{i \downarrow},
$$

where $c_{i \sigma}^{\dagger}\left(c_{i \sigma}\right)$ is a creation (annihilation) operator of spin up/down $(\sigma=\uparrow, \downarrow)$ electrons on lattice site $i, n_{i \sigma}=c_{i \sigma}^{\dagger} c_{i \sigma}$, and $t(U)$ denotes the nearest-neighbor hopping (on-site repulsion). This is known to be a model Hamiltonian for graphene with $U / t \approx 3^{11}$. More importantly, it is not geometrically frustrated, namely, as seen in Fig. 1, the neighboring sites of any site on A sublattice belong to B sublattice (and vice versa). Indeed, it is well known that the ground state becomes an antiferromagnetic insulator (AFMI), i.e., classically Néel ordered, from a semi-metal (SM) with increasing $U / t^{12}$.

Very recently, Meng et al. ${ }^{2}$ has reexamined the ground state phase diagram of this model and found a possible spin liquid phase in the range $3.4 \leqq U / t \leqq 4.3$ between SM and AFMI. Their finding is rather surprising because it is widely believed that a stable spin liquid occurs most likely in frustrated quantum systems where strong quantum fluctuations destroy the long-range magnetic order even at zero temperature ${ }^{1}$. Their study was particularly successful because, with the auxiliary field technique ${ }^{13}$, there is no sign problem in the corresponding quantum Monte Carlo simulations, and an accurate finite size scaling was possible by using numerically exact 


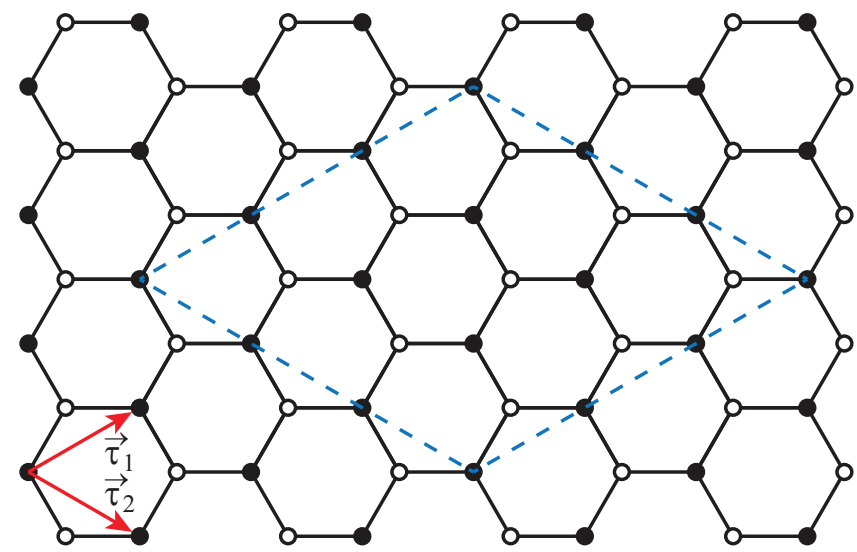

Figure $1 \mid$ The honeycomb lattice. Primitive lattice vectors $\vec{\tau}_{1}$ and $\vec{\tau}_{2}$ are denoted by red arrows. As an example, the honeycomb lattice with $L=3$ is indicated by dashed blue lines. Solid and open circles indicate sites on A and B sublattices, respectively.

results for clusters containing up to 648 sites. So far, their results represent the most important numerical evidence for a possible spin liquid ground state in a "realistic" electronic model in two dimensions (2D), because, to our knowledge, only a particularly simplified quantum dimer model on the triangular lattice ${ }^{9}$ and the Kitaev model ${ }^{10}$, built $a d$ hoc to have an exact solution, allow a spin liquid ground state in 2D. Furthermore, their results were considered to be a clear violation of the "Murphy's Law": in a too simple model, not vexed by the "fermion sign problem", nothing interesting can occur ${ }^{5}$.

Here, by performing simulations for much larger clusters containing up to 2592 sites, we show that antiferromagnetic order concomitantly occurs once the insulating behavior sets in, supporting the more conventional Hartree-Fock (HF) transition from SM to $\mathrm{AFMI}^{12}$. Although our results agree with the previous study for the same clusters up to 648 sites, we have reached a quite different conclusion, as the possible spin liquid region reduces substantially to a small interval $3.8 t \leqq U / t \leqq 3.9 t$, if it ever exists. This reminds us similar claims on spin liquid behaviors in different systems in $2 \mathrm{D}^{14,15}$, which have been corrected later on by much larger cluster simulations, showing instead antiferromagnetic long-range order ${ }^{16,17}$.

\section{Results}

We use finite size clusters of $N=2 L^{2}$ sites (thus containing $L \times L$ unit cells) with periodic boundary conditions (see Fig. 1), which satisfy all symmetries of the infinite lattice ${ }^{18}$ (also see Supplementary information). Here $L$ is the linear dimension of the cluster and we take $L$ up to 36 . We use the well established auxiliary field Monte Carlo technique ${ }^{13}$, which allows the statistical evaluation of the following quantity,

$$
O(\tau)=\frac{\left\langle\psi_{L}\left|e^{-\frac{\tau}{2} \hat{H}} \hat{O} \mathrm{e}^{-\frac{\tau}{2} \hat{H}}\right| \psi_{R}\right\rangle}{\left\langle\psi_{L}\left|\mathrm{e}^{-\tau \hat{H}}\right| \psi_{R}\right\rangle},
$$

where $\hat{O}$ is a physical operator, $\left|\psi_{R}\right\rangle\left(\left|\psi_{L}\right\rangle\right)$ is the right (left) trial wave function (not orthogonal to the exact ground state), and $\tau$ is the projection time. The exact ground state expectation value $\langle\hat{O}\rangle$ of
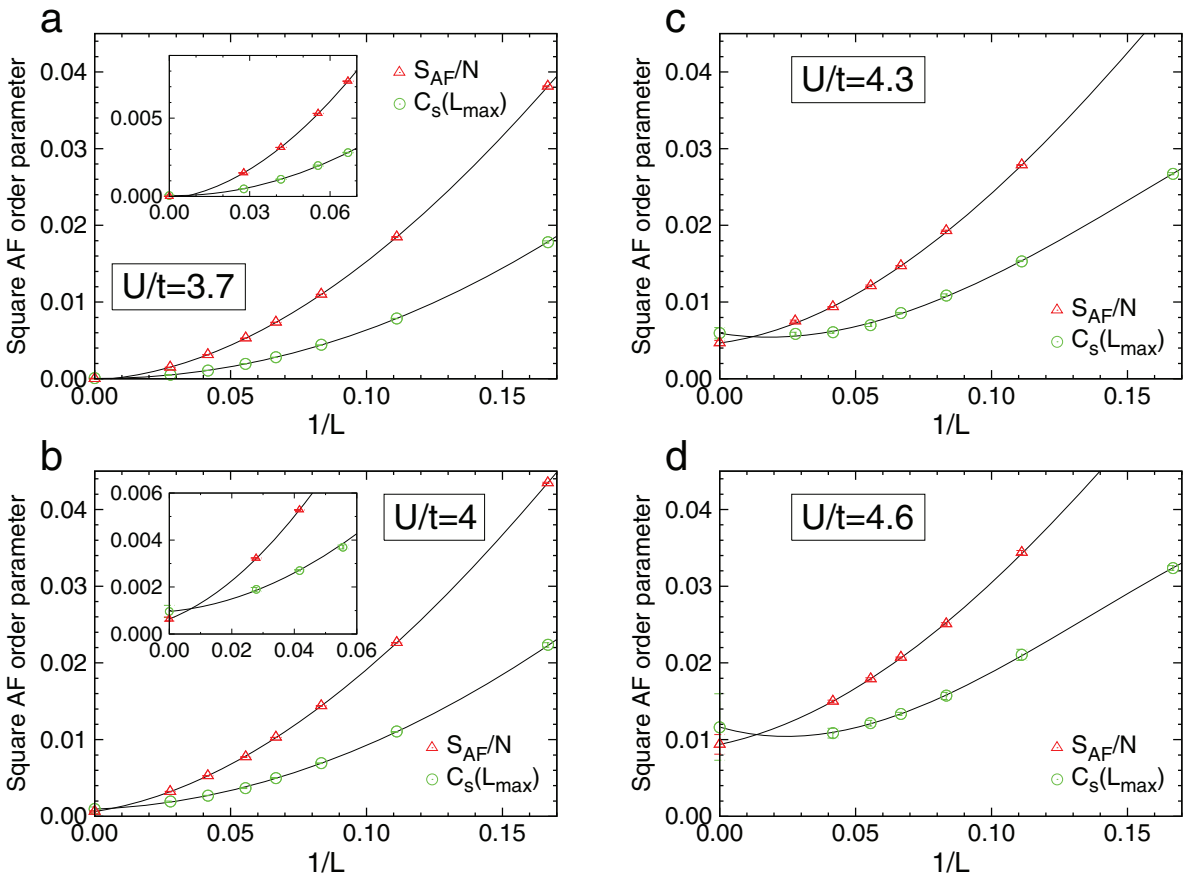

Figure $2 \mid$ Finite size scaling of spin-spin correlation functions for the Hubbard model on the honeycomb lattice at half-filling. Spin structure factor, $S_{\mathrm{AF}}$, and spin-spin correlations at the maximum distance, $C_{s}\left(L_{\mathrm{max}}\right)$, are denoted by triangles and circles, respectively. Here, $L$ is the linear size of clusters containing $N=2 L^{2}$ sites. Antiferromagnetic order parameter squared, $m_{s}^{2}$, is estimated by finite size extrapolating $S_{\mathrm{AF}}$ and $C_{s}\left(L_{\mathrm{max}}\right)$ to $L \rightarrow \infty$, namely, $m_{s}^{2}=\lim _{L \rightarrow \infty} S_{\mathrm{AF}} / N=\lim _{L \rightarrow \infty} C_{s}\left(L_{\mathrm{max}}\right)$. Solid curves are fit of the data by cubic polynomials in $1 / L$. It is clearly seen that a consistent extrapolated value $m_{s}^{2}$ is obtained for both quantities $S_{\mathrm{AF}}$ and $C_{s}\left(L_{\mathrm{max}}\right)$, indicated respectively by triangles and circles at $1 / L=0$. Error bars of the extrapolated values are computed with a resampling technique described in Methods. Insets show the expanded plots for large $L$. The fits are stable upon removal of the data for the largest (i.e., $L=36$ ) or the smallest (i.e., $L=6$ ) size, and the extrapolated value of $C_{s}\left(L_{\max }\right)$ is always consistent with $S_{A F} / N$ within two standard deviations. Including the largest size calculations in the fits increases the extrapolated values slightly and at the same time gives more consistent values of $C_{s}\left(L_{\max }\right)$ and $S_{A F} / N$ in $L \rightarrow \infty$, thus clearly indicating that our present estimate provides an accurate lower bound for the AF order parameter $m_{s}$. All data presented in this figure refers to $\Delta \tau t=0.1$, because the Trotter $\Delta \tau$ error is essentially negligible (see Fig. 3). More details are found in Supplementary information. 


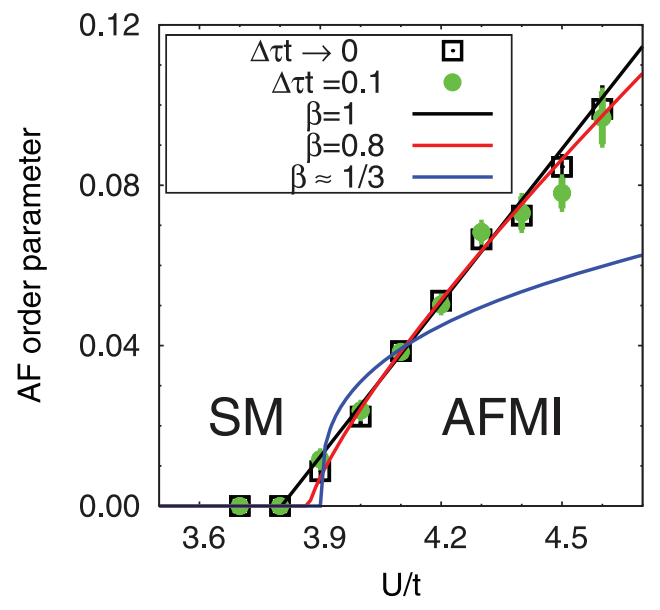

Figure $3 \mid$ The ground state phase diagram for the half-filled Hubbard model on the honeycomb lattice. Antiferromagnetic order parameter $m_{s}$ (open squares) as a function of $U / t$. The error, due to the finite $\Delta \tau$ in the evaluation of $S_{A F}$, is removed by quadratic extrapolations for $\Delta \tau t=0.1$, $\Delta \tau t=0.2$, and $\Delta \tau t=0.4$ (see Supplementary information for details). The antiferromagnetic order parameter $m_{S}$ is obtained by finite-size extrapolating the square root of $S_{A F} / N, m_{s}=\lim _{L \rightarrow \infty} \sqrt{S_{A F} / N}$, as shown in Fig. 2. For comparison, $m_{s}$ estimated by finite-size extrapolating $S_{A F}$ for $\Delta \tau t$ $=0.1$ without the $\Delta \tau$ correction is also plotted (solid circles). SM and AFMI stand for semi-metal and antiferromagnetic insulator, respectively. Solid lines are fit of $m_{s}$ with the critical behavior $m_{s}=\left(U_{c}-U\right)^{\beta}$, for selected critical exponents $\beta . \beta=1$ for the HF theory ${ }^{12}, \beta=0.3362$ for the classical critical theory of quantum magnets ${ }^{19}$, and $\beta=0.80 \pm 0.04$ is the best fit of our data. In any case, the critical $U_{c}$ ranges from $U_{c} / t=3.8(\beta=$ 1) to $U_{c} / t=3.9(\beta=0.3362)$. Our best estimate is $U_{c} / t=3.869 \pm 0.013$.

the operator $\hat{O}$ is then obtained by adopting the limit of $\tau \rightarrow \infty$ and $\Delta \tau$ $\rightarrow 0$ for $O(\tau)$, where $\Delta \tau$ is the short time discretization of $\tau$. This approximation - the so called Trotter approximation - is necessary to introduce the auxiliary fields ${ }^{13}$ and implies a systematic error, negligible for small $\Delta \tau$ (see Supplementary information).

First, we study both the spin structure factor $S_{\mathrm{AF}}=\frac{1}{N}\left\langle\left[\sum_{r}\left(S_{r, \mathrm{~A}}\right.\right.\right.$ $\left.\left.\left.-S_{r, \mathrm{~B}}\right)\right]^{2}\right\rangle$ and the spin-spin correlations $C_{s}(R)=\left\langle S_{r, \mathrm{~A}} \cdot S_{r+\mathrm{R}, \mathrm{A}}\right\rangle$ at the maximum distance $|R|=L_{\max }$ of each cluster for $U / t=4$, where the strongest evidence of a spin liquid behavior was found in Ref. 2. Here $S_{r, \mathrm{~A}}\left(S_{r} \mathrm{~B}\right)$ is the spin operator at unit cell $\boldsymbol{r}$ on A (B) sublattice. As shown in Fig. 2b, our results show consistently a finite value of the antiferromagnetic order parameter $m_{s}^{2}=S_{\mathrm{AF}} / N=C\left(L_{\max }\right)$ for $L \rightarrow$ $\infty$, in sharp contrast to the existence of a spin liquid, i.e., spin disordered, ground state reported in Ref. 2.

By doing similar calculations for several $U / t$ values (see Fig. 2 and Supplementary information), we find in Fig. 3 that $m_{s}$ approximately scales linearly with respect to $U / t$, i.e., $m_{s} \propto\left|U-U_{c}\right|^{\beta}$, with a critical exponent $\beta \simeq 0.8$, which is similar to the critical behavior $(\beta=1)$ predicted by the HF theory ${ }^{12}$. Although corrections to this almost linear critical behavior are obviously expected, they do not change much the critical value $U_{c}$ at which the antiferromagnetic order melts, as clearly shown in Fig. 3. Our best estimate of the critical value is $U_{c} / t=3.869 \pm 0.013$, which is much smaller than the one $(\approx$ 4.3) reported in Ref. 2 . Note, however, that the critical exponent $\beta$ may be different from the present estimate if the critical region is very close to $U_{c}$. In such case the accurate determination of $\beta$ obviously requires much larger clusters which are not feasible at present.

Let us now evaluate the spin gap $\Delta_{s}$. In order to avoid possible errors in extrapolating the imaginary time displaced spin-spin correlation functions, here we calculate directly the total energies in the singlet and the triplet sectors, with improved estimators, which dramatically reduce their statistical errors ${ }^{20}$ (also see Supplementary information). We can see clearly in Fig. 4a that the extrapolated spin gaps for different $U / t$ values are always zero within statistical errors (e.g., the statistical error as small as $0.004 t$ for $U / t=4$ ).

Next, we investigate whether the system is metallic or insulating, namely, whether there exists a zero or a finite charge gap. For this purpose, it is enough to simply study the long distance behavior of charge-charge correlations, $\rho(\boldsymbol{R})=\left\langle n_{r, \mathrm{~A}} n_{\boldsymbol{r}+\boldsymbol{R}, \mathrm{A}}\right\rangle-\left\langle n_{r, \mathrm{~A}}\right\rangle\left\langle n_{r+\boldsymbol{R}, \mathrm{A}}\right\rangle$.
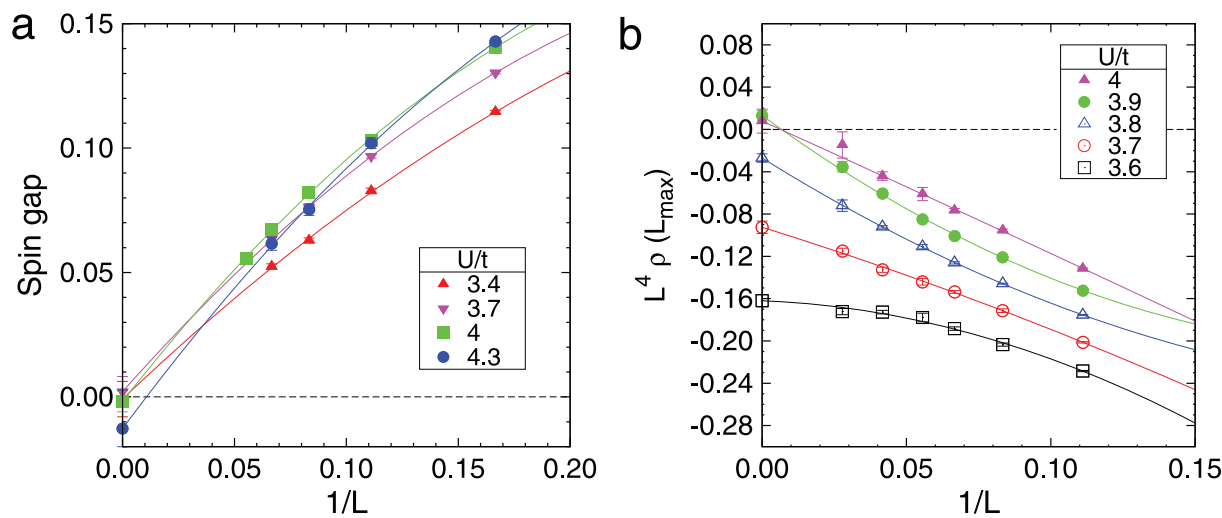

Figure $4 \mid$ Finite size scaling of spin gap and charge-charge correlation functions for the Hubbard model on the honeycomb lattice at half-filling. (a) Spin gap $\Delta_{s}=E(S=1)-E(S=0)$ for various $U / t$, where $E(S)$ is the lowest energy for a given spin $S$. Solid curves are fits of data by quadratic polynomials in $1 / L$. The extrapolated values are also indicated at $1 / L=0$. Error bars of the extrapolated values are computed with the resampling technique. In the semi-metallic region, the spin gap scales to zero with increasing the resolution in momentum space, namely as $1 / L$. In the antiferromagnetic region, the spin gap should instead vanish as $1 / L^{2}$. This explains why for $U / t=4.3$ the gap extrapolates to negative values, as we are well inside the antiferromagnetic phase (see Fig. 3). In any case, a sizable spin gap is not found for any value of $U / t$. (b) Charge-charge correlation function $\rho(\boldsymbol{R})=\left\langle n_{r, \mathrm{~A}} n_{r+R, \mathrm{~A}}\right\rangle-\left\langle n_{r, \mathrm{~A}}\right\rangle\left\langle n_{r+R, \mathrm{~A}}\right\rangle$ at the maximum distance $|\boldsymbol{R}|=L_{\max }$ for several values of $U / t$. In the semi-metallic phase, $\rho(R) \sim 1 / R^{4}$ and $L^{4} \rho\left(L_{\mathrm{max}}\right)$ should converge to a finite value for $L \rightarrow \infty$. Instead, when a charge gap opens, the charge-charge correlations should decay exponentially and $L^{4} \rho\left(L_{\text {max }}\right)$ converges to zero in this limit. Indeed, a quadratic extrapolation to $L \rightarrow \infty$ of this quantity, which is clearly appropriate in the semi-metallic phase, appears to vanish in the interval between $U / t=3.8$ and $U / t=3.9$, in remarkable agreement with the critical value $U_{c}=3.869 \pm 0.013$ estimated for the antiferromagnetic transition (see Fig. 3). Obviously, a polynomial fit is not consistent in the insulating region and this explains why the extrapolated value to $1 / L=0$ seems slightly positive in this case. For the spin gap and the charge-charge correlation functions, the Trotter $\Delta \tau$ error is negligible, and all data shown here refers to $\Delta \tau t=0.14$ and 0.1 , respectively. 
Here $n_{r, A}$ is the density operator at unit cell $\boldsymbol{r}$ on A sublattice (see Fig 1). They should change from power law to exponential behavior at a critical $U$ where the charge gap opens up. This change of behavior is evident in Fig. $4 \mathrm{~b}$ and appears consistently around the onset of the antiferromagnetic transition $\left(U_{c}\right)$, within a remarkably small uncertainty $<0.1 t$ on the value of $U$. Our results therefore strongly support the more conventional scenario of a direct and continuous quantum phase transition between SM and AFMI ${ }^{12}$.

\section{Discussion}

Let us now discuss here why we have not found any evidence of a spin liquid phase. As shown in Ref. 21, by applying one of the theorems by $\mathrm{Lieb}^{22}$, it is easily proved that the exact ground state of this model for $U \neq 0$ satisfies the Marshall sign rule ${ }^{23}$ in the sector of no doubly occupied sites, accounting for low energy spin excitations. Indeed, the phases coincide with those of the simple antiferromagnetic Néel state ordered along the $x$-spin quantization axis, $\prod_{R \in A}\left(|\uparrow\rangle_{R}-|\downarrow\rangle_{R}\right) \prod_{R \in B}$ $\left(|\uparrow\rangle_{R}+|\downarrow\rangle_{R}\right)$, where $|\uparrow\rangle_{R}$ and $|\downarrow\rangle_{R}$ are spin configurations (along the $z$-spin quantization axis) at site $R$. The expansion of this state in terms of $|\uparrow\rangle_{R}$ and $|\downarrow\rangle_{R}$ yields the simple Marshall sign, namely, it is negative if the number of spin down in the A sublattice is odd. Thus, the phases of the ground state are trivial in the bosonic spin $1 / 2$ sector. Therefore, Bose-Einstein condensation can hardly be avoided and a magnetic long-range order occurs once the charge gap becomes finite.

At this point, one could be tempted to assume the general validity of the above observation for generic $S=1 / 2$ model Hamiltonians with $\mathrm{SU}(2)$ invariance and use this criteria based on the phases of the ground state wave function as a powerful guideline in the search of spin liquids for model systems as well as for real materials. Indeed, in all unfrustrated spin-1/2 Heisenberg and Hubbard models in the sector of no doubly occupied sites, the phases of the ground state wave function are not at all entangled in real space as they factorize into independent contributions relative to each site. Instead, the phases of the ground state wave functions are highly non trivial in well established spin liquid models such as, for instance, the Kitaev's model $^{10}$, and the celebrated quantum dimer model on the triangular lattice ${ }^{9}$, because they are described by paired wave functions, which couple in a non trivial way the phases of nearest neighbor spins ${ }^{24}$.

Therefore, we conclude that in a true spin liquid in $2 \mathrm{D}$, the phases of the ground state wave function should be highly non trivial and entangled, otherwise any seed of spin liquid behavior would be most likely destabilized. To our best knowledge, the above observation is valid so far for all spin-1/2 models with $S U(2)$ invariance. Notice that the restriction to $\mathrm{SU}(2)$ invariant models appears to be important because the spin-1/2 easy-axis Heisenberg model on the Kagome lattice most likely display spin liquid behavior ${ }^{25-27}$. Here, however, the calculations have not been confirmed on fairly low temperatures yet. Therefore, further numerical study is required for understanding what are the key ingredients that stabilize a spin liquid phase in "realistic" electronic models.

\section{Methods}

Here we employ the standard auxiliary field Monte Carlo algorithm ${ }^{13}$ with a more efficient implementation ${ }^{20}$ by using different left and right trial functions $\left|\psi_{L}\right\rangle$ and $\left|\psi_{R}\right\rangle$ in equation (2). We include also in the trial wave function a Gutzwiller type projection, $\exp \left(-g \Sigma_{i} n_{i \uparrow} n_{i \downarrow}\right)$, where $g$ is the Gutzwiller variational parameter, to optimize the efficiency. As reported in Ref. 20, the statistical error in evaluating the energy $E(S)$ for a given spin $S$ is dramatically reduced for appropriate values of $g$. Thus we can evaluate the spin gap $\Delta_{S}=E(S=1)-E(S=0)$ with high accuracy, without facing the negative sign problem, by directly simulating the singlet $S=0$ and the triplet $S=1$ sectors separately (see Supplementary information).

In order to accelerate the convergence to the ground state, we use for $\left|\psi_{R}\right\rangle$ a Slater determinant with a definite spin $S$, by breaking only spatial symmetries to remove the degeneracy at momenta $K$ and $K^{\prime}$ for the clusters chosen (a similar strategy was adopted in Ref. 2). Conversely, we use for $\left|\psi_{L}\right\rangle$ a rotational and translationally invariant Slater determinant obtained by diagonalizing a mean field Hamiltonian, containing an explicit antiferromagnetic order parameter directed along the $x$-spin quantization axis. In this way, the left and the right trial wave functions break different symmetries (spin and spatial ones, respectively), and for any symmetric operator $\hat{O}$ the convergence to the ground state is expected to be much faster because it is dominated by the singlet gap in the symmetric sector $\Delta_{\text {gap }}$, that is clearly much larger than, e.g., the lowest triplet excitation in the magnetic phase. Since $\Delta_{\text {gap }}$ is expected to scale to zero (if indeed zero) at most as $\simeq 1 / L$, we use a projection time $\tau=(L+4) / t$, which we have tested carefully to be large enough for well converged results (see Supplementary information). We have also checked that the systematic error due to discretizing $\tau$ is basically negligible with $\Delta \tau t=0.14$ for the spin gap calculations and with $\Delta \tau t=0.1$ for the correlation functions (see Fig. 3 and Supplementary information)

In order to evaluate the statistical errors of the finite size extrapolations, we use a straightforward resampling technique. This resampling technique is used, for example, when values of $S_{A F}$ calculated for finite sizes are extrapolated to $L \rightarrow \infty$ to estimate $m_{s}$ in Fig. 3. Let us denote in general the calculated Monte Carlo data $f(L)$ and the corresponding statistical error $\delta f(L)$ obtained for a cluster of size $L$. The main goal of this resampling technique is to estimate the finite-size extrapolated value $c_{0}$ and its statistical error $\delta c_{0}$ when the Monte Carlo data are fitted by, e.g., cubic polynomials,

$$
f(L)=\sum_{n=0}^{3} \frac{c_{n}}{L^{n}} .
$$

In this resampling technique, we first generate for each $L$ a "fictitious sample" which is normally distributed around $f(L)$ with its standard deviation $\delta f(L)$, which is also an output of the quantum Monte Carlo simulation. Then, we fit these "fictitious" data to equation (3), by using the weighted (with $1 /(\delta f(L))^{2}$ ) least square fit, and estimate $c_{0}$. We repeat this $M_{\mathrm{rs}}$ times so that we have now $M_{\mathrm{rs}}$ samples for $\mathcal{c}_{0}$, i.e.,

$\left\{c_{0}^{(1)}, c_{0}^{(2)}, \ldots, c_{0}^{\left(M_{\mathrm{rs}}\right)}\right\}$ distributed according to a probability distribution consistent with the Monte Carlo simulations. Finally, we simply average $\left\{c_{0}^{(i)}\right\}\left(i=1,2, \ldots, M_{\mathrm{rs}}\right)$ for $\left\langle c_{0}\right\rangle=\lim _{L \rightarrow \infty} f(L)$, and the standard deviation of $\left\{c_{0}^{(i)}\right\}$ gives an estimate of the statistical error $\delta c_{0}$ of the extrapolated value $\left\langle c_{0}\right\rangle$. We take $M_{\mathrm{rs}}=200$. We have checked that the resultant $\left\langle c_{0}\right\rangle$ and $\delta c_{0}$ are not dependent on $M_{\mathrm{rs}}$ as long as $M_{\mathrm{rs}}$ is large enough.

1. Balents, L. Spin liquids in frustrated magnets. Nature 464, 199-208 (2010).

2. Meng, Z. Y., Lang, T. C., Wessel, S., Assaad, F. F. \& Muramatsu, A. quantum spin liquid emerging in two-dimensional correlated Dirac fermions. Nature 464, 847-851 (2010)

3. Yan, S., Huse, D. A. \& White, S. R. Spin-liquid ground state of the $S=1 / 2$ Kagome Heisenberg antiferromagnet. Science 332, 1173-1176 (2011).

4. Jiang, H.-C., Yao, H. \& Balents, L. Spin liquid ground state of the spin-1/2 square $J_{1}-J_{2}$ Heisenberg model. Phys. Rev. B 86, 024424 (2012).

5. Kivelson, S. A. Spin liquid ground states? http://www.condmatjournalclub.org/ $? \mathrm{p}=1251(2011)$.

6. Shimizu, Y., Miyagawa, K., Kanoda, K., Maesato, M. \& Saito, G. Spin liquid state in an organic Mott insulator with a triagngular lattice. Phys. Rev. Lett. 91, 107001 (2003).

7. Kurosaki, Y., Shimizu, Y., Miyagawa, K., Kanoda, K. \& Saito, G. Mott transition from s spin liquid to a Fermi liquid in the spin-frustrated organic conductor $\kappa-(\mathrm{ET})_{2} \mathrm{Cu}_{2}(\mathrm{CN})_{3}$. Phys. Rev. Lett. 95, 177001 (2005).

8. Itou, T., Oyamada, A., Maegawa, S., Tamura, M. \& Kato, R. Quantum spin liquid in the spin-1/2 triangular antiferromagnet $\mathrm{EtMe}_{3} \mathrm{Sb}\left[\mathrm{Pd}(\mathrm{dmit})_{2}\right]_{2}$. Phys. Rev. B 77, 104413 (2008).

9. Fendley, P., Moessner, R. \& Sondhi, S. L. Classical dimers on the triangular lattice. Phys. Rev. B 66, 214513 (2002).

10. Kitaev, A. Anyons in an exactly solved model and beyond. Annals of Physics 321, 2-111 (2006).

11. Castro Neto, A. H., Guinea, F., Peres, N. M. R., Novoselov, K. S. \& Geim, A. K. The electronic properties of graphene. Rev. Mod. Phys. 81, 109-162 (2009).

12. Sorella, S. \& Tosatti, E. Semimetal-insulator transition of the Hubbard model in the honeycomb lattice. Europhys. Lett. 19, 699-704 (1992).

13. Hirsch, J. E. Two dimensional Hubbard model: numerical simulation study. Phys. Rev. B 31, 4403-4419 (1985).

14. Santoro, G., Sorella, S., Guidoni, L., Parola, A. \& Tosatti, E. Spin-liquid ground state in a two-dimensional nonfrustrated spin model. Phys. Rev. Lett. 83, 3065-3068 (1999).

15. Parola, A., Sorella, S. \& Zhong, Q. F. Realization of a spin liquid in a two dimensional quantum antiferromagnet. Phys. Rev. Lett. 71, 4393-4396 (1993).

16. Harada, K., Kawashima, N. \& Troyer, M. Néel and spin-Peierls ground states of two-dimensional $\mathrm{SU}(N)$ quantum antiferromagnets. Phys. Rev. Lett. 90, 117203 (2003).

17. Sandvik, A. W. Multichain mean-field theory of quasi-one-dimensional quantum spin systems. Phys. Rev. Lett. 83, 3069-3072 (1999).

18. Bernu, B., Lecheminant, P., Lhuillier, C. \& Pierre, L. Exact spectra, spin susceptibilities, and order parameter of the quantum Heisenberg antiferromagnet on the triangular lattice. Phys. Rev. B 50, 10048-10062 (1994).

19. Guida, R. \& Zinn-Justin, J. Critical exponents of the N-vector model. J. Phys. A 31, 8103-8121 (1998).

20. Hlubina, R., Sorella, S. \& Guinea, F. Ferromagnetism in the two dimensional t-t Hubbard model at the Van Hove density. Phys. Rev. Lett. 78, 1343-1346 (1997). 
21. Li, T. Absence of topological degeneracy in the Hubbard model on honeycomb lattice. Europhys. Lett. 93, 37007 (2011).

22. Lieb, E. H. Two theorems on the Hubbard model. Phys. Rev. Lett. 62, 1201-1204 (1989).

23. Marshall, W. Antiferromagnetism. Proc. R. Soc. London Ser. A 232, 48-68 (1955)

24. Yunoki, S. \& Sorella, S. Two spin liquid phases in the spatially anisotropic triangular Heisenberg model. Phys. Rev. B 74, 014408 (2006).

25. Balents, L., Fisher, M. P. A. \& Girvin, S. M. Fractionalization in an easy-axis Kagome antiferromagnet. Phys. Rev. B 65, 224412 (2002).

26. Isakov, S. V., Hastings, M. B. \& Melko, R. G. Topological entanglement entropy of a Bose-Hubbard spin liquid. Nature Physics 7, 772-775 (2011)

27. Isakov, S. V., Melko, R. G. \& Hastings, M. B. Universal signatures of fractionalized quantum critical points. Science 13, 193-195 (2012).

\section{Acknowledgments}

We acknowledge E. Tosatti, F. Becca, and T. Li for useful discussions. We are also grateful to A. Muramatsu and F. F. Assaad for valuable comments and providing us some of their numerical data reported in Ref. 2. This work is supported by a PRACE grant 2010PA0447 and by MIUR-COFIN2012. Part of the results is obtained by the K computer at RIKEN Advanced Institute for Computational Science.

\section{Author contributions}

S.S. developed the numerical codes; all authors performed the numerical simulations, analyzed the numerical data, and wrote the manuscript.

\section{Additional information}

Supplementary information accompanies this paper at http://www.nature.com/ scientificreports

Competing financial interests: The authors declare no competing financial interests.

License: This work is licensed under a Creative Commons

Attribution-NonCommercial-NoDerivs 3.0 Unported License. To view a copy of this license, visit http://creativecommons.org/licenses/by-nc-nd/3.0/

How to cite this article: Sorella, S., Otsuka, Y. \& Yunoki, S. Absence of a Spin Liquid Phase in the Hubbard Model on the Honeycomb Lattice. Sci. Rep. 2, 992; DOI:10.1038/srep00992 (2012) 